\title{
Evaluation of Automated Segmentation Algorithm for Macular Volumetric Measurements of Eight Individual Retinal Layer Thickness
}

\author{
Ori Zahavi, Alberto Domínguez-Vicent*, Rune Brautaset (D) and Abinaya Priya Venkataraman
}

check for updates

Citation: Zahavi, O.;

Domínguez-Vicent, A.; Brautaset, R.;

Venkataraman, A.P. Evaluation of

Automated Segmentation Algorithm for Macular Volumetric

Measurements of Eight Individual Retinal Layer Thickness. Appl. Sci. 2021, 11, 1250. https://doi.org/ 10.3390/app11031250

\section{Academic Editor:}

Miguel Souto-Bayarri

Received: 7 January 2021

Accepted: 27 January 2021

Published: 29 January 2021

Publisher's Note: MDPI stays neutral with regard to jurisdictional claims in published maps and institutional affiliations.

Copyright: (c) 2021 by the authors. Licensee MDPI, Basel, Switzerland. This article is an open access article distributed under the terms and conditions of the Creative Commons Attribution (CC BY) license (https:// creativecommons.org/licenses/by/ $4.0 /)$.
Division of Eye and Vision, Department of Clinical Neuroscience, Karolinska Institute, 17177 Stockholm, Sweden; ori.zahavi@ki.se (O.Z.); rune.brautaset@ki.se (R.B.); abinaya.venkataraman@ki.se (A.P.V.)

* Correspondence: alberto.dominguez.vicent@ki.se; Tel.: +46-700915398; Fax: +46-86723846

\begin{abstract}
Background: We evaluated the performance of an automated algorithm available on a clinical OCT (Canon-HS100) for macular volumetric measurements of eight individual retinal layers. Methods and Analysis: Two consecutive three-dimensional scans were acquired on 29 subjects with healthy retinas. Thickness measurements were obtained from eight individual retinal layers in nine macular sectors based on Early Treatment Diabetic Retinopathy Study (ETDRS) protocol. The repeatability was evaluated using the within-subject standard deviation from which the repeatability limits (Rlimit) and coefficient of variation $(\mathrm{CoV})$ were calculated. Results: The repeatability metrics varied among different layers and sectors. The variation among the sectors was larger in two of the outer layers (plexiform and nuclear layer) and the retinal nerve fiber layer. For the other five layers, the repeatability limit was less than $5 \mu \mathrm{m}$ and $\mathrm{CoV}$ was less than $7.5 \%$ in all nine ETDRS sectors. Conclusions: The repeatability of the OCT-HS100 to measure eight individual retinal layers is good in general. Nevertheless, the repeatability is not homogeneous among different layers and sectors. This needs to be taken into account while designing clinical measurement protocols.
\end{abstract}

Keywords: layer segmentation; macular layers; optical coherence tomography; repeatability; retinal thickness

\section{Introduction}

Optical Coherence Tomography (OCT) is the only clinical equipment that allows the in-vivo visualization of individual layers of the retina [1]. This plays an important role in the diagnosis and monitoring of various ocular and neurological diseases [2-5]. Different types of OCT are available commercially and each type comes with its own automated segmentation algorithm [6]. The objective and quantitative information provided by the OCT is valuable in the diagnosis and follow up of patients with glaucoma [7], macular diseases $[4,8]$, and neurological disorders $[9,10]$. The segmentation algorithms available for the clinical OCT instruments provide data, mainly the total retinal thickness and individual layer thickness of ganglion cell layer (GCL) and retinal nerve fiber layer (RNFL).

Recent developments in instrumentation and image analysis algorithms allow further segmentation of individual layers, which is of great use in different areas in ophthalmology and neurology. Changes in inner retinal layers such as the GCL and RNFL are shown to be a hallmark in the diagnosis of glaucoma $[2,11]$. The thickness of the inner retinal layers is reported to change in different neurologic disorders including multiple sclerosis $[9,12]$ and Parkinson's disease $[13,14]$. There are other retinal disorders like central serous chorioretinopathy, age-related macular degeneration, and inherited retinal dystrophies that cause changes in outer retinal layers [15-19]. Most clinical OCTs report volumetric information of individual retinal layer thickness of specific layers like RNFL and GCL [20-22]. Due to the increased use of OCT in the clinical practice for the evaluation of the individual layer thickness, it is important to have reliable inbuilt segmentation software. 
The reliability of the OCT's inbuilt segmentation algorithms for retinal thickness measurement and individual layer thickness measurement of RNFL and GCL have been widely studied in the past [20-23]. The thickness measurement in these layers has been shown to be clinically acceptable in general. The individual retinal layer thickness assessed using external segmentation algorithms has also been reported to be reliable [24,25]. As the field is developing, new OCT instruments with better imaging resolution and improved segmentation algorithms are developed continuously, which allows assessing individual layer thickness in a larger retinal area. As these instruments are made available for clinical use, there is an on-going need to assess the precision of the new OCTs and the new algorithms in both healthy eyes and in eyes with different pathologies. This study evaluates the repeatability of the OCT-HS100 (Canon, Tokyo, Japan) for the macular volumetric thickness measurements of eight different retinal layers.

\section{Methods and Materials}

\subsection{Participants}

Twenty-nine subjects participated in the study and all subjects underwent a comprehensive eye health evaluation including intraocular pressure measurement and retinal examination. The mean age of the participants was $35 \pm 13$ years and had no media opacities, retinal disorders, prior ocular surgeries or trauma. The study adhered to the tenets of the Declaration of Helsinki and was approved by the Swedish Ethical Review Authority. A written informed consent was obtained from the study participants after the nature and purpose of the study had been clearly explained.

\subsection{OCT Scans}

Two OCT scans on macula were performed with Canon OCT-HS100 (version 4.00) on the right eye of the participants without the use of any mydriatic drops. The Canon OCT-HS100 has an A-scan velocity of 70,000 scans/sec with an axial resolution of $3 \mu \mathrm{m}$. A three-dimensional scan protocol composed of 1024 A-scans for each of the horizontally oriented 128 B-scans was used for macular thickness measurements. The scans were centered on fovea and covered an area of $10 \times 10 \mathrm{~mm}$. The scans were repeated if there was a blink during the measurement or if the signal strength was less than 7 . The OCT instrument uses retinal tracking for eye movements during the scanning procedure to minimize artifacts.

The automated segmentation software of the instrument was used to delineate eight different layers of the retina: Retinal Nerve Fiber Layer (RNFL), Ganglion Cell Layer (GCL), Inner Plexiform Layer (IPL), Inner Nuclear Layer (INL), Outer Plexiform Layer (OPL), Outer Nuclear Layer (ONL), Outer Segment (OS), and Retinal Pigment Epithelium (RPE). No manual adjustment of the segmentation was performed. The thickness of each layer specified above was evaluated in nine sectors based on Early Treatment Diabetic Retinopathy Study (ETDRS) protocol with the central circle, inner ring (Superior, Nasal, Inferior and temporal), and outer ring (Superior, Nasal, Inferior and temporal) with diameters of 1 , 3 , and $6 \mathrm{~mm}$, respectively. Figure 1 shows an example of individual layer segmentation (panel A) and the schematic representation of the ETDRS sectors (panel B).

\subsection{Statistical Analysis}

The metrics used to describe the repeatability of individual layer thickness measurement were the within subject standard deviation (Sw), repeatability limits (Rlimit), and coefficient of variation (CoV). The Sw, which represents the repeatability of the measurements, was calculated with a one-way analysis of variance with the subject as a factor. Rlimit was calculated as $1.96 \sqrt{ } 2 \mathrm{Sw}$, and it represents the expected limits that $95 \%$ of the measurements should be within [26]. CoV is calculated as Sw divided by average thickness of that particular layer and was expressed in percentage. The sample size required to estimate the individual retinal thickness with the precision as close to the axial resolution 
$(3 \mu \mathrm{m})$ of the OCT for 2 repeated measurements was calculated to be 27 subjects [27]. This calculation was based on an assumption that the individual layer can be as thin as $10 \mu \mathrm{m}$.
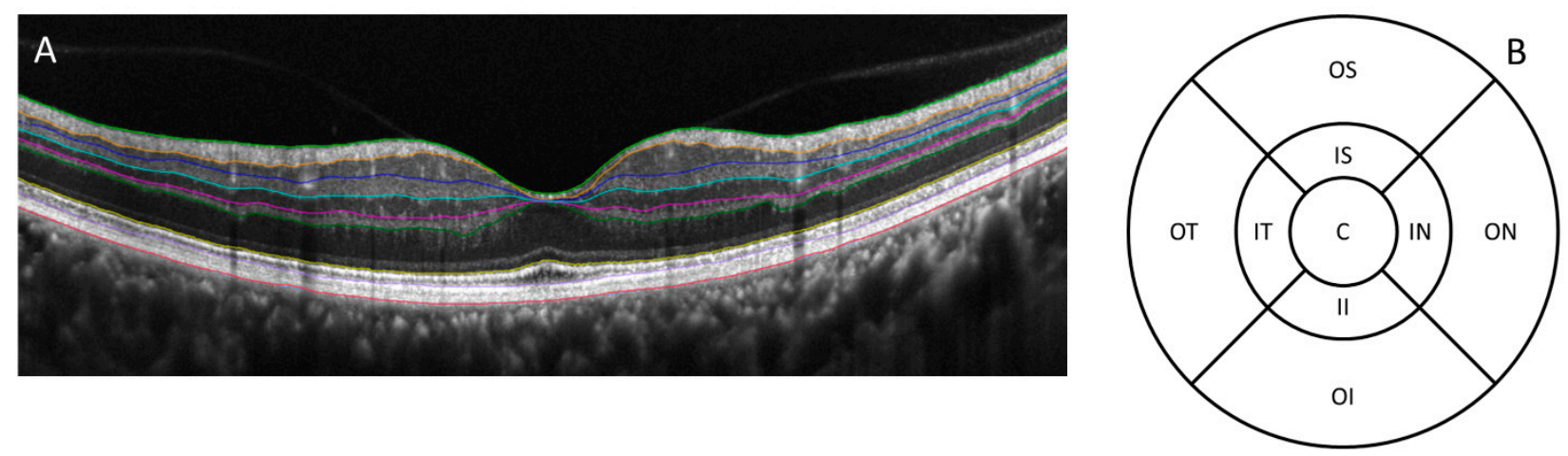

Figure 1. Example of the individual layer segmentation on a single B-scan (panel A) and a schematic representation of the ETDRS sector map (panel B). C: Central, IS: Inner Superior, OS: Outer Superior, IN: Inner Nasal, ON: Outer Nasal, II: Inner Inferior, OI: Outer Inferior, IT: Inner Temporal, OT: Outer Temporal.

\section{Results}

The descriptive statistics of each retinal layer in the ETDRS sectors are shown in Table 1. On average, the thickness in the central circle was more than that of the surrounding circles for ONL, OS, and RPE. The opposite tendency was seen for the other five layers. Comparing inner and outer circles, the RNFL was thicker in the outer circle than in the inner circle, except for the temporal sector where no such tendency was seen. For all the other layers except OS, the inner circle was thicker than the outer circle. The thickness was similar in the inner and outer circle for OS.

Table 1. Thickness values of the eight retinal layers in the ETDRS macular sectors.

\begin{tabular}{|c|c|c|c|c|c|c|c|c|c|}
\hline & Central & $\begin{array}{c}\text { Inner } \\
\text { Infe- } \\
\text { rior }\end{array}$ & $\begin{array}{c}\text { Outer } \\
\text { Infe- } \\
\text { rior }\end{array}$ & $\begin{array}{l}\text { Inner } \\
\text { Nasal }\end{array}$ & $\begin{array}{l}\text { Outer } \\
\text { Nasal }\end{array}$ & $\begin{array}{c}\text { Inner } \\
\text { Supe- } \\
\text { rior }\end{array}$ & $\begin{array}{c}\text { Outer } \\
\text { Supe- } \\
\text { rior }\end{array}$ & $\begin{array}{l}\text { Inner } \\
\text { Tem- } \\
\text { poral }\end{array}$ & $\begin{array}{c}\text { Outer } \\
\text { Tem- } \\
\text { poral }\end{array}$ \\
\hline RNFL & $11 \pm 6$ & $27 \pm 3$ & $40 \pm 5$ & $23 \pm 6$ & $\begin{array}{c}50 \pm \\
4.9\end{array}$ & $25 \pm 3$ & $39 \pm 5$ & $19 \pm 5$ & $18 \pm 1$ \\
\hline GCL & $17 \pm 4$ & $51 \pm 5$ & $27 \pm 3$ & $53 \pm 5$ & $32 \pm 4$ & $52 \pm 4$ & $30 \pm 3$ & $48 \pm 5$ & $32 \pm 3$ \\
\hline IPL & $16 \pm 3$ & $42 \pm 3$ & $33 \pm 3$ & $41 \pm 3$ & $35 \pm 4$ & $41 \pm 3$ & $33 \pm 3$ & $38 \pm 3$ & $36 \pm 3$ \\
\hline INL & $18 \pm 3$ & $44 \pm 2$ & $32 \pm 3$ & $44 \pm 3$ & $35 \pm 3$ & $43 \pm 2$ & $34 \pm 3$ & $39 \pm 2$ & $35 \pm 2$ \\
\hline OPL & $10 \pm 5$ & $35 \pm 8$ & $27 \pm 4$ & $36 \pm 9$ & $30 \pm 4$ & $33 \pm 5$ & $26 \pm 2$ & $28 \pm 3$ & $25 \pm 2$ \\
\hline ONL & $\begin{array}{c}122 \pm \\
10\end{array}$ & $\begin{array}{c}83 \pm \\
12\end{array}$ & $69 \pm 6$ & $\begin{array}{c}89 \pm \\
13\end{array}$ & $73 \pm 8$ & $91 \pm 9$ & $80 \pm 7$ & $98 \pm 8$ & $79 \pm 6$ \\
\hline OS & $35 \pm 1$ & $29 \pm 3$ & $31 \pm 3$ & $29 \pm 2$ & $29 \pm 3$ & $29 \pm 2$ & $30 \pm 4$ & $29 \pm 2$ & $30 \pm 4$ \\
\hline RPE & $37 \pm 2$ & $34 \pm 3$ & $28 \pm 3$ & $34 \pm 3$ & $31 \pm 3$ & $35 \pm 3$ & $31 \pm 4$ & $35 \pm 2$ & $32 \pm 4$ \\
\hline
\end{tabular}

The values represent average \pm 1 standard deviation. All values are expressed in microns. RNFL: Retinal nerve fiber layer, GCL: Ganglion cell layer, IPL: Inner plexiform layer, INL: Inner nuclear layer, OPL: Outer plexiform layer, ONL: Outer nuclear layer, OS: Outer segment, RPE: Retinal pigment epithelium.

Figure 2 shows the Rlimit values of the measurements for each retinal layer in each sector of the macular ETDRS map. In five out of eight layers (GCL, IPL, INL, OS, and RPE), the Rlimit was less than $5 \mu \mathrm{m}$ in all the ETDRS sectors. For each of these five layers, the maximum difference in Rlimit among the sectors was $4 \mu \mathrm{m}$. For OPL, the Rlimit was below $10 \mu \mathrm{m}$ for all the sectors and the maximum difference among them was $7 \mu \mathrm{m}$. Both RNFL and ONL had some sectors with Rlimit exceeding $10 \mu \mathrm{m}$ (RNFL-inner nasal sector and 
ONL-Central, inner superior, and inner inferior sectors). The maximum difference in Rlimit among the sectors for RNFL and ONL were 11 and $10 \mu \mathrm{m}$, respectively.

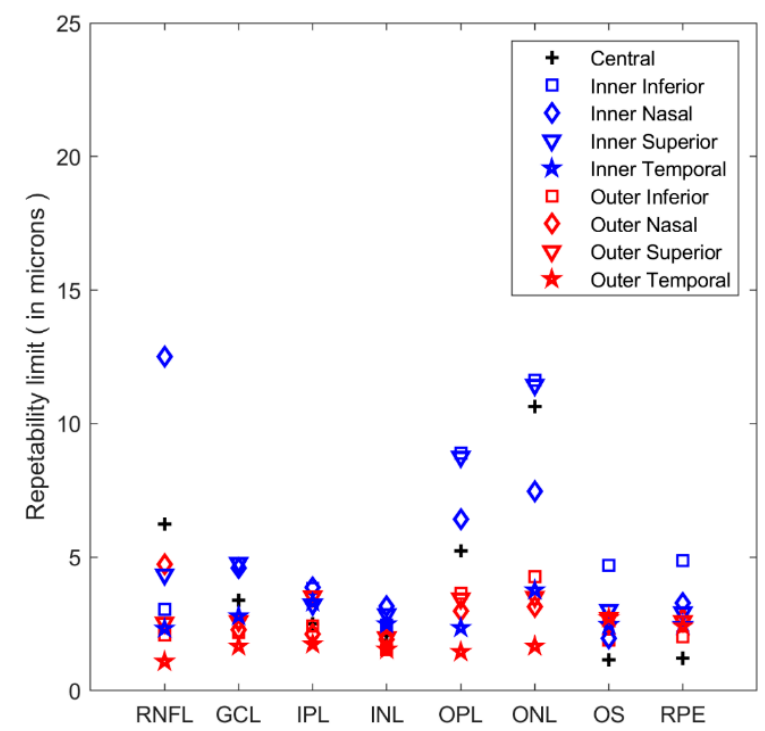

Figure 2. Repeatability limit values of the measurements for each retinal layer in each sector of the ETDRS map. Retinal Nerve Fiber Layer (RNFL), Ganglion Cell Layer (GCL), Inner Plexiform Layer (IPL), Inner Nuclear Layer (INL), Outer Plexiform Layer (OPL), Outer Nuclear Layer (ONL), Outer Segment (OS), and Retinal Pigment Epithelium (RPE).

The relationship between the $\mathrm{Sw}$ and the $\mathrm{CoV}$ for the eight individual layers is shown in Figure 3. The Sw of the GCL, IPL, INL, OS, and RPE layers was under $2 \mu \mathrm{m}$ and the $\mathrm{CoV}$ was below 7.5\%. In GCL, IPL, and INL, the central sector had the largest CoV though the Sw was similar to the other sectors, whereas the central sector in OS and RPE showed the best $\mathrm{Sw}$ (around $0.5 \mu \mathrm{m}$ ) and CoV (around 1\%). For the other three layers (RNFL, OPL, and $\mathrm{ONL}$ ), the $\mathrm{Sw}$ values had a larger spread among the sectors, though the maximum $\mathrm{Sw}$ value was about $4.5 \mu \mathrm{m}$. For these layers, the least variability in $\mathrm{CoV}$ among the sectors was seen for ONL with the maximum $\mathrm{CoV}$ of $5 \%$. For both OPL and ONL, the central and inner sectors except the inner temporal sector had larger Sw compared to the outer sectors (OS and RPE). The RNFL showed the largest variability in CoV among the sectors with the maximum $\mathrm{CoV}$ of $21 \%$. In RNFL, except for the inner nasal sector, the other sectors showed smaller and similar Sw values (around $2.5 \mu \mathrm{m}$ ). However, the CoV of RNFL in both the central and the inner nasal sector was almost three times larger compared to the other sectors. 

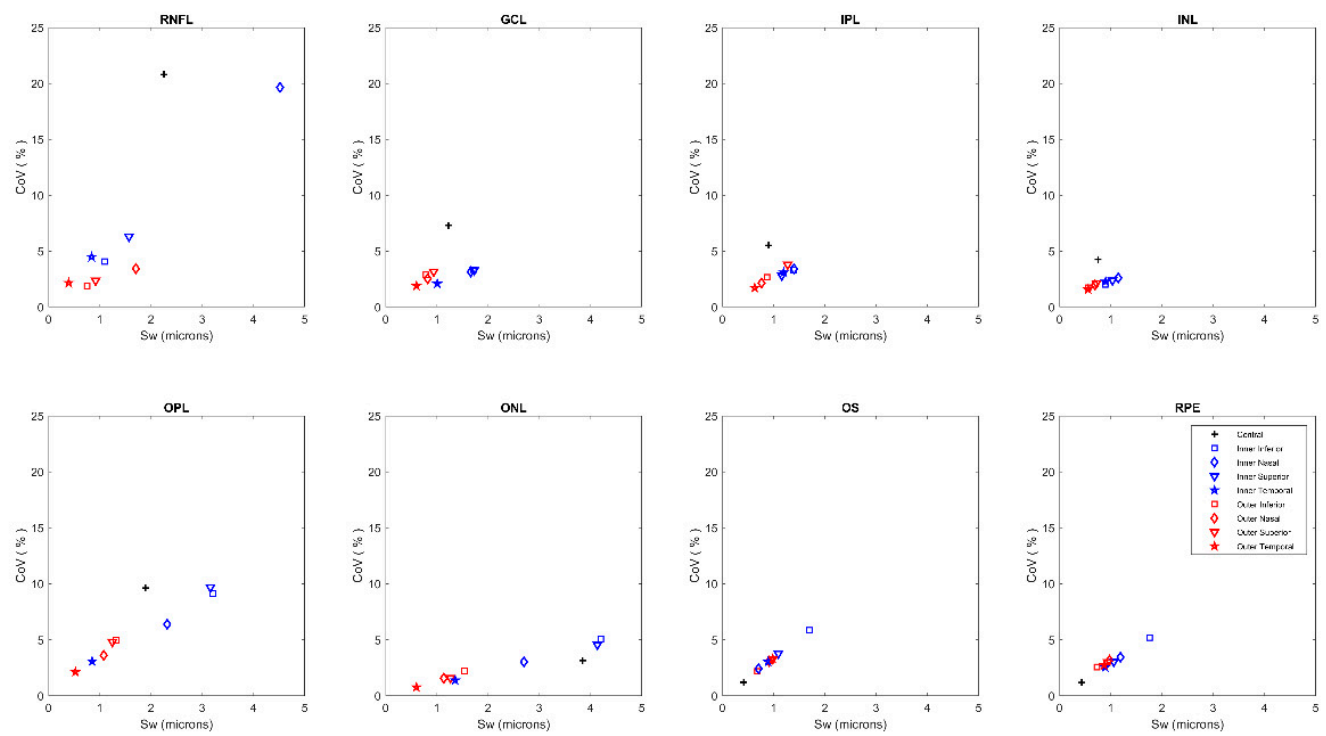

Figure 3. Relationship between the within subject standard deviation and the Coefficient of variation (CoV). Sw: Within subject standard deviation, Retinal Nerve Fiber Layer (RNFL), Ganglion Cell Layer (GCL), Inner Plexiform Layer (IPL), Inner Nuclear Layer (INL), Outer Plexiform Layer (OPL), Outer Nuclear Layer (ONL), Outer Segment (OS), and Retinal Pigment Epithelium (RPE).

\section{Discussion}

This study assessed the performance of the segmentation algorithm of the OCT-HS100 to measure the thickness of eight individual retinal layers. The thickness was measured by automated layer segmentation software in nine ETDRS sectors. We show that there are variations in the repeatability both among the different sectors and the different layers. However, the repeatability was good in general for all eight retinal layers evaluated.

The average thickness values of the individual retinal layers and its variability among the subjects reported in the present study are on a similar range to that of previously reported values $[28,29]$. The repeatability of thickness measurements of RNFL and GCLIPL complexes have been reported extensively in many of the previous studies $[6,20-23,28]$. In the present study, we have evaluated the GCL and IPL layers separately and also evaluated the other individual layers in different ETDRS sectors. In general, there was some difference in the repeatability between the outer and inner sectors. One possible reason for this difference could be that the area of the inner sectors is much smaller than the outer sectors. When the segmentation software calculates the average thickness within the sector, any segmentation error will have a bigger impact on inner sectors than outer sectors. The present study used horizontal B-scans, and this could also have an impact on the repeatability of the different sectors [22]. The presence of blood vessels in the superior and inferior sectors can result in segmentation artefacts, and this could affect the mean thickness value of these sectors. The effect can also be more for inner sectors than outer sectors due to smaller area. In central sector, the repeatability varied between the different retinal layers. The best Sw was seen for OS and RPE and the worst for ONL.

All of the inner retinal layers and OPL had a larger CoV in the central sector. The variability in the central sector is expected due to the thinner layers in that central sector. The variability for OPL could be due to the angle of the OCT's light beam. Oberwahrenbrock et al. [28] also reported that OPL had the worst repeatability. The CoV is inversely related to the actual thickness and hence, even if the Sw was smaller, the CoV can be larger. Larger variations in the repeatability among the sectors are seen in RNFL, OPL, and ONL. For RNFL, this variation can be due to the difference in the thickness among the sectors and that it is also the thinnest layer. For OPL and ONL, the variation could be mainly due to the difficulty in segmentation. The OPL contains both the photoreceptor synopses and Henle fiber layer. Depending upon the angle of the incident light from OCT, Henle fiber 
layer can appear either as a hyper-reflective or as a hypo-reflective structure [28,30-33]. As a consequence, the delineation of the OPL and ONL layers can be affected. Another report also shows that the agreement in the measurement values between horizontal and vertical scans are particularly bad for OPL and ONL layers [34].

The three most outer layers in the retina (RPE, OS and ONL) are thicker in the central sector and uniformly thinner in the other sectors. This makes it easier for the segmentation algorithm to identify and delineate these layers. The repeatability for RPE and OS is good as expected, but, for the ONL, the repeatability varies among the sectors. As mentioned earlier, the repeatability of ONL can be affected due to the difficulties of the segmentation algorithm in identifying and delineating the OPL-ONL layer.

We can estimate the minimum number of measurements needed to ensure a measurement tolerance (MT) that is the same as the instrument's axial resolution, using the expression for MT, $(M T=(1.96 \cdot S w) / \sqrt{ } N)$ for $\mathrm{N}$ number of measurements according to the ISO standards $[35,36]$. Based on the present results, for the measurement of GCL, IPL, INL, OS, and RPE in the ETDRS sectors, only one measurement is enough. Based on the sector that had the worst repeatability, a larger number of measurements will be needed for RNFL, OPL and ONL (9, 4, and 8, respectively). However, based on the sector with second worst repeatability, the number of measurements needed for these layers reduce significantly to 2, 2, and 6, respectively. The higher number of measurements needed for ONL reflects the difficulties in segmenting this layer as discussed earlier. For pathologies where the measurement of ONL is important, we need to take this factor into consideration while planning the measurement protocol.

Reliability of the quantitative measurement from OCT is very important as these measurements are used for the screening, diagnosis, and follow up of various retinal and neural disorders. OCT has been shown to be useful in detecting and monitoring changes in individual retinal layer thickness in diseases like Glaucoma [7], age related macular degeneration [4,37], diabetic retinopathy [8], inherited retinal disorders [16,38], and neurological disorders $[5,10,13]$. Knowledge on the repeatability of OCT for individual retinal layer measurements is important for clinicians. This is needed to differentiate if the observed changes in the retinal thickness are due to measurement fluctuations or actual pathological changes. The limitation of the present study is that only healthy eyes were analyzed. For disease follow up, we need to know the repeatability on pathologic eyes, and further studies on individual layer thickness repeatability are recommended.

\section{Conclusions}

The repeatability of the OCT-HS100 to measure the eight different retinal layers is good. In most of the ETDRS sectors, only one or two OCT 3-D scans are needed to get a measurement tolerance, the same as the axial resolution of the instrument for the individual retinal layers except for the outer nuclear layer.

Author Contributions: Conceptualization: O.Z., A.D.-V., R.B., A.P.V.; Methodology: O.Z., A.D.-V., A.P.V.; Formal analysis: A.D.-V., A.P.V.; Writing-Original draft preparation: O.Z., A.D.-V., A.P.V.; Writing-Review and editing: O.Z., A.D.-V., R.B., A.P.V.; Supervision: A.D.-V., R.B., A.P.V. All authors have read and agreed to the published version of the manuscript.

Funding: This research was funded by promoting vision research fund from Ögönfonden (Sweden), grant number: 2019.

Institutional Review Board Statement: The study protocol adhered to the tenets of the Declaration of Helsinki and was approved by the Regional Ethical Committee (Regionala etikpröningsnämden, Stockholm 2011/874-31/2).

Informed Consent Statement: Informed consent was obtained from all subjects involved in the study.

Data Availability Statement: All data is included in the manuscript.

Acknowledgments: This study was supported in part by the Ögönfonden (Sweden). 
Conflicts of Interest: The authors declare no conflict of interest.

\section{References}

1. Thomas, D.; Duguid, G. Optical coherence tomography-A review of the principles and contemporary uses in retinal investigation. Eye 2004, 18, 561-570. [CrossRef] [PubMed]

2. Mwanza, J.-C.; Budenz, D.L. New developments in optical coherence tomography imaging for glaucoma. Curr. Opin. Ophthalmol. 2018, 29, 121-129. [CrossRef] [PubMed]

3. Sung, K.R.; Wollstein, G.; Kim, N.R.; Na, J.H.; Nevins, J.E.; Kim, C.Y.; Schuman, J.S. Macular assessment using optical coherence tomography for glaucoma diagnosis. Br. J. Ophthalmol. 2012, 96, 1452-1455. [CrossRef]

4. Castillo, M.M.; Mowatt, G.; Elders, A.; Lois, N.; Fraser, C.; Hernández, R.; Amoaku, W.; Burr, J.M.; Lotery, A.; Ramsay, C.R.; et al. Optical coherence tomography for the monitoring of neovascular age-related macular degeneration: A systematic review. Ophthalmology 2015, 122, 399-406. [CrossRef] [PubMed]

5. Petzold, A.; de Boer, J.F.; Schippling, S.; Vermersch, P.; Kardon, R.; Green, A.; Calabresi, P.A.; Polman, C. Optical coherence tomography in multiple sclerosis: A systematic review and meta-analysis. Lancet Neurol. 2010, 9, 921-932. [CrossRef]

6. Pierro, L.; Gagliardi, M.; Iuliano, L.; Ambrosi, A.; Bandello, F. Retinal nerve fiber layer thickness reproducibility using seven different OCT instruments. Investig. Ophthalmol. Vis. Sci. 2012, 53, 5912-5920. [CrossRef] [PubMed]

7. Kansal, V.; Armstrong, J.J.; Pintwala, R.; Hutnik, C. Optical coherence tomography for glaucoma diagnosis: An evidence based meta-analysis. PLoS ONE 2018, 13, e0190621. [CrossRef]

8. De Clerck, E.E.B.; Schouten, J.S.A.G.; Berendschot, T.T.J.M.; Kessels, A.G.H.; Nuijts, R.M.M.A.; Beckers, H.J.M.; Schram, M.T.; Stehouwer, C.D.A.; Webers, C.A.B. New ophthalmologic imaging techniques for detection and monitoring of neurodegenerative changes in diabetes: A systematic review. Lancet Diabetes Endocrinol. 2015, 3, 653-663. [CrossRef]

9. Saidha, S.; Al-Louzi, O.; Ratchford, J.N.; Bhargava, P.; Oh, J.; Newsome, S.D.; Prince, J.L.; Pham, D.; Roy, S.; van Zijl, P.; et al. Optical coherence tomography reflects brain atrophy in multiple sclerosis: A four-year study. Ann. Neurol. 2015, 78, 801-813. [CrossRef]

10. Graham, E.C.; You, Y.; Yiannikas, C.; Garrick, R.; Parratt, J.; Barnett, M.H.; Klistorner, A. Progressive Loss of Retinal Ganglion Cells and Axons in Nonoptic Neuritis Eyes in Multiple Sclerosis: A Longitudinal Optical Coherence Tomography Study. Investig. Ophthalmol. Vis. Sci. 2016, 57, 2311-2317. [CrossRef]

11. Chen, T.C.; Hoguet, A.; Junk, A.K.; Nouri-Mahdavi, K.; Radhakrishnan, S.; Takusagawa, H.L.; Chen, P.P. Spectral-Domain OCT: Helping the Clinician Diagnose Glaucoma: A Report by the American Academy of Ophthalmology. Ophthalmology 2018, 125, 1817-1827. [CrossRef] [PubMed]

12. Birkeldh, U.; Manouchehrinia, A.; Hietala, M.A.; Hillert, J.; Olsson, T.; Piehl, F.; Kockum, I.S.; Brundin, L.; Zahavi, O.; WahlbergRamsay, M.; et al. The Temporal Retinal Nerve Fiber Layer Thickness Is the Most Important Optical Coherence Tomography Estimate in Multiple Sclerosis. Front. Neurol. 2017, 8, 675. [CrossRef] [PubMed]

13. Bayhan, H.A.; Aslan Bayhan, S.; Tanık, N.; Gürdal, C. The association of spectral-domain optical coherence tomography determined ganglion cell complex parameters and disease severity in Parkinson's disease. Curr. Eye Res. 2014, 39, 1117-1122 [CrossRef] [PubMed]

14. Lee, J.-Y.; Ahn, J.; Kim, T.W.; Jeon, B.S. Optical coherence tomography in Parkinson's disease: Is the retina a biomarker? J. Parkinsons Dis. 2014, 4, 197-204. [CrossRef]

15. Tao, L.W.; Wu, Z.; Guymer, R.H.; Luu, C.D. Ellipsoid zone on optical coherence tomography: A review. Clin. Experiment. Ophthalmol. 2016, 44, 422-430. [CrossRef]

16. Hood, D.C.; Zhang, X.; Ramachandran, R.; Talamini, C.L.; Raza, A.; Greenberg, J.P.; Sherman, J.; Tsang, S.H.; Birch, D.G. The inner segment/outer segment border seen on optical coherence tomography is less intense in patients with diminished cone function. Investig. Ophthalmol. Vis. Sci. 2011, 52, 9703-9709. [CrossRef]

17. Spaide, R.F. Outer retinal atrophy after regression of subretinal drusenoid deposits as a newly recognized form of late age-related macular degeneration. Retina 2013, 33, 1800-1808. [CrossRef]

18. Matsumoto, H.; Sato, T.; Kishi, S. Outer nuclear layer thickness at the fovea determines visual outcomes in resolved central serous chorioretinopathy. Am. J. Ophthalmol. 2009, 148, 105-110. [CrossRef]

19. Lamin, A.; Oakley, J.D.; Dubis, A.M.; Russakoff, D.B.; Sivaprasad, S. Changes in volume of various retinal layers over time in early and intermediate age-related macular degeneration. Eye 2019, 33, 428-434. [CrossRef]

20. Matlach, J.; Wagner, M.; Malzahn, U.; Gobel, W. Repeatability of peripapillary retinal nerve fiber layer and inner retinal thickness among two spectral domain optical coherence tomography devices. Investig. Ophthalmol. Vis. Sci. 2014, 55, 6536-6546. [CrossRef]

21. Toteberg-Harms, M.; Sturm, V.; Knecht, P.B.; Funk, J.; Menke, M.N. Repeatability of nerve fiber layer thickness measurements in patients with glaucoma and without glaucoma using spectral-domain and time-domain OCT. Graefes. Arch. Clin. Exp. Ophthalmol. 2012, 250, 279-287. [CrossRef] [PubMed]

22. Domínguez-Vicent, A.; Brautaset, R.; Venkataraman, A.P. Repeatability of quantitative measurements of retinal layers with SD-OCT and agreement between vertical and horizontal scan protocols in healthy eyes. PLoS ONE 2019, 14, e0221466. [CrossRef] [PubMed]

23. Ctori, I.; Huntjens, B. Repeatability of Foveal Measurements Using Spectralis Optical Coherence Tomography Segmentation Software. PLoS ONE 2015, 10, e0129005. [CrossRef] [PubMed] 
24. Liu, X.; Shen, M.; Huang, S.; Leng, L.; Zhu, D.; Lu, F. Repeatability and reproducibility of eight macular intra-retinal layer thicknesses determined by an automated segmentation algorithm using two SD-OCT instruments. PLoS ONE 2014, 9, e87996. [CrossRef] [PubMed]

25. Terry, L.; Cassels, N.; Lu, K.; Acton, J.H.; Margrain, T.H.; North, R.V.; Fergusson, J.; White, N.; Wood, A. Automated Retinal Layer Segmentation Using Spectral Domain Optical Coherence Tomography: Evaluation of Inter-Session Repeatability and Agreement between Devices. PLoS ONE 2016, 11, e0162001. [CrossRef] [PubMed]

26. McAlinden, C.; Khadka, J.; Pesudovs, K. Statistical methods for conducting agreement (comparison of clinical tests) and precision (repeatability or reproducibility) studies in optometry and ophthalmology. Ophthalmic Physiol. Opt. 2011, 31, 330-338. [CrossRef]

27. McAlinden, C.; Khadka, J.; Pesudovs, K. Precision (repeatability and reproducibility) studies and sample-size calculation. J. Cataract Refract. Surg. 2015, 41, 2598-2604. [CrossRef]

28. Oberwahrenbrock, T.; Weinhold, M.; Mikolajczak, J.; Zimmermann, H.; Paul, F.; Beckers, I.; Brandt, A.U. Reliability of Intra-Retinal Layer Thickness Estimates. PLoS ONE 2015, 10, e0137316. [CrossRef]

29. Kim, J.H.; Lee, S.H.; Han, J.Y.; Kang, H.G.; Byeon, S.H.; Kim, S.S.; Koh, H.J.; Lee, S.C.; Ki, M. Comparison of Individual Retinal Layer Thicknesses between Highly Myopic Eyes and Normal Control Eyes Using Retinal Layer Segmentation Analysis. Sci. Rep. 2019, 9, 14000. [CrossRef]

30. Otani, T.; Yamaguchi, Y.; Kishi, S. Improved visualization of Henle fiber layer by changing the measurement beam angle on optical coherence tomography. Retina 2011, 31, 497-501. [CrossRef]

31. Ouyang, Y.; Walsh, A.C.; Keane, P.A.; Heussen, F.M.; Pappuru, R.K.R.; Sadda, S.R. Different phenotypes of the appearance of the outer plexiform layer on optical coherence tomography. Graefe's Arch. Clin. Exp. Ophthalmol. Albr. von Graefes Arch. fur Klin. und Exp. Ophthalmol. 2013, 251, 2311-2317. [CrossRef] [PubMed]

32. Lujan, B.J.; Roorda, A.; Knighton, R.W.; Carroll, J. Revealing Henle's fiber layer using spectral domain optical coherence tomography. Investig. Ophthalmol. Vis. Sci. 2011, 52, 1486-1492. [CrossRef] [PubMed]

33. Mrejen, S.; Gallego-Pinazo, R.; Freund, K.B.; Paques, M. Recognition of Henle's fiber layer on OCT images. Ophthalmology 2013, 120, e32-e33. [CrossRef] [PubMed]

34. Gonzalez Caldito, N.; Antony, B.; He, Y.; Lang, A.; Nguyen, J.; Rothman, A.; Ogbuokiri, E.; Avornu, A.; Balcer, L.; Frohman, E.; et al. Analysis of Agreement of Retinal-Layer Thickness Measures Derived from the Segmentation of Horizontal and Vertical Spectralis OCT Macular Scans. Curr. Eye Res. 2018, 43, 415-423. [CrossRef] [PubMed]

35. ISO Probability and General Statistical Terms. Statistics: Vocabulary and symbols. Int. Organ. Stand. 2006. ISO 3534-1:2006.

36. ISO Accuracy (trueness and precision) of measurement methods and results—Part 1: General principles and definitions. Int. Organ. Stand. 1994. ISO 5725-1:1994.

37. Muftuoglu, I.K.; Ramkumar, H.L.; Bartsch, D.-U.; Meshi, A.; Gaber, R.; Freeman, W.R. Quantitative analysis of the inner retinal layer thicknesses in age-related macular degeneration using corrected optical coherence tomography segmentation. Retina 2018, 38, 1478-1484. [CrossRef]

38. Witkin, A.J.; Ko, T.H.; Fujimoto, J.G.; Chan, A.; Drexler, W.; Schuman, J.S.; Reichel, E.; Duker, J.S. Ultra-high resolution optical coherence tomography assessment of photoreceptors in retinitis pigmentosa and related diseases. Am. J. Ophthalmol. 2006, 142, 945-952. [CrossRef] 